EDITORIAL

\title{
El empoderamiento en el ámbito de la gerontología clínica y social
}

\section{Empowerment in a social and clinical gerontology context}

\author{
Raúl Vaca Bermejo ${ }^{a}$, Pilar Monreal-Bosch ${ }^{\mathrm{b}}$, Lourdes Bermejo García ${ }^{\mathrm{c}, *}$, Yolanda Cotiello Cueria ${ }^{\mathrm{d}}$, \\ Sandra Fernández Prado ${ }^{\mathrm{e}}$, María Rosario Limón Mendizábal ${ }^{\mathrm{f}}$, Xavier Lorente Guerrerog ${ }^{\mathrm{g}}$, \\ María del Carmen Benítez Ramírez ${ }^{\mathrm{h}}$, Elena López Romero ${ }^{\mathrm{i}}$ y Ana Rodriguez Valcarce ${ }^{\mathrm{j}, 1}$ \\ a Asociación Española de Psicogerontología Barcelona, Bardelona, España \\ ${ }^{\mathrm{b}}$ Grupo de investigación ECIS, Universitat de Girona, Girona, España \\ ${ }^{\mathrm{c}}$ Consultora Profesional Autónoma, Santander, Santander, España \\ d Consultorio Salud Venta las Ranas, Villaviciosa, Asturias, España \\ e Centro de Día Alzheimer AGADEA, Ribeira, Coruña, España \\ ${ }^{\mathrm{f}}$ Facultad de Educación, Universidad Complutense de Madrid, Madrid, España \\ ${ }^{g}$ Facultad de Ciencias Sociales y de la Comunicación, Universidad Católica San Antonio de Murcia, Murcia, España

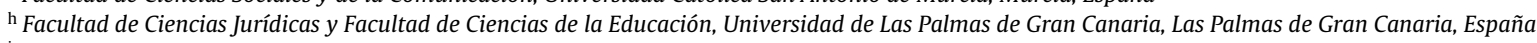 \\ i Barcelona, España \\ j Hospital Santa Clotilde, Santander, España
}

El empoderamiento es el proceso mediante el que las personas fortalecen capacidades, confianza, visión y protagonismo en cuanto que forman parte de un grupo social, para impulsar cambios positivos en las situaciones en las que viven. Está directamente vinculado a la promoción de la persona, a su desarrollo integral, al ejercicio de sus derechos y a su calidad de vida. Persigue así, aumentar el poder personal, social y/o político de modo que los individuos, las familias y las comunidades puedan actuar para mejorar sus situaciones vitales. Es por ello que, cada vez más, el «empoderamiento» es considerado una meta fundamental en áreas tales como la intervención psicosocial o las actividades de promoción, prevención e intervención en salud ${ }^{1}$ y hablar de empoderamiento requiere referirse tanto al empoderamiento de las personas (esfera individual, ya sean usuarios o profesionales), en los recursos (esfera organizacional) y al de los grupos sociales (esfera comunitaria ${ }^{2-4}$ ).

Empoderar implica también crear capacidades. Desde hace unos años existe un movimiento mundial conocido como el enfoque del desarrollo humano o "de las capacidades" ${ }^{5}$ que impulsa un nuevo paradigma teórico en el campo del desarrollo y las políticas públicas. Este enfoque concibe a cada persona como un fin en sí misma y no se pregunta solamente por su grado de bienestar, sino también por las oportunidades de elección y libertad disponibles para cada ser humano. La finalidad del desarrollo global consiste en hacer posible que las personas vivan vidas plenas y creativas,

\footnotetext{
* Autor para correspondencia.

Correo electrónico: lourdesbermejo@telefonica.net (L. Bermejo García).

1 Todos los autores son miembros del Grupo de Trabajo Interdisciplinar de Educación Gerontológica de la SEGG
}

desarrollen su potencial y formen una existencia significativa acorde con la igualdad de dignidad humana de todos los individuos.

En el ámbito de la gerontología existe actualmente una clara tendencia por promover el empoderamiento de las personas mayores (PM) en el cuidado y mantenimiento de su estado de salud y de su bienestar. Este proceso persigue desechar los modelos que fijan su foco de atención en las limitaciones y problemas de las PM por otros centrados en el desarrollo de sus potencialidades y fortalezas personales.

En esta línea se vincula a promover y defender la autonomía y participación activa y efectiva de las PM en la gestión de los diferentes aspectos que conciernen directamente con su vida, además de los relacionados con el cuidado de su salud. Este es un aspecto especialmente delicado en el ámbito de estas personas, ya que se trata de un colectivo potencialmente vulnerable compuesto por personas con características y vivencias personales muy diversas que pueden dificultar los procesos de empoderamiento: problemas de salud, disminución de sus competencias personales, pérdida de apoyos naturales, menor nivel de autoconciencia de sus derechos, necesidades psicoafectivas no satisfechas, menor nivel educativo con diferentes culturas, sentimientos de soledad, etc. Esta situación tiene como consecuencia el aumento de una visión estereotipada y estigmatizada y acentuar la tendencia a ignorar la voluntad de las PM y su capacidad para ejercer control sobre los aspectos más centrales de su vida actual ${ }^{1}$.

Entre las PM y los profesionales se establece una relación interpersonal con connotaciones éticas, filosóficas y sociológicas de tipo profesional que sirve de base a la gestión de la salud y bienestar de la persona ${ }^{6}$. Esta relación, hoy en día, está inmersa en un proceso de cambio que evoluciona desde una concepción y actitud más 
paternalista de los profesionales hacia una de mayor preponderancia de la autodeterminación o autonomía de la persona ${ }^{7}$. La tendencia es aumentar el empoderamiento de las PM para que participen en el proceso de toma de decisiones sobre los aspectos que afectan directamente a su vida cotidiana y su salud con el objetivo de mejorar su calidad de vida.

Los profesionales que trabajan con PM han de regirse por criterios y adoptar decisiones orientadas a potenciar su empoderamiento $^{8}$. Es necesario conocer, valorar y contar con la capacidad cognitiva y educativa de cada persona, así como con su voluntad para conseguir un mayor nivel de bienestar y salud.

El proceso de empoderamiento dirige a las PM hacia un desarrollo saludable buscando la realización plena de sus potencialidades. Se basa en la idea de que las PM tienen mayor éxito en su vida cuando identifican y usan sus fortalezas, habilidades y activos; y también, asume que los seres humanos tienen la capacidad de crecer y cambiar ${ }^{9}$. Esta perspectiva proporciona una estructura y un contexto para evaluar objetivos realistas, movilizar los recursos para promover el cambio y la autoestima e infundir esperanza en el futuro.

Los profesionales que trabajan desde esta perspectiva están comprometidos con los valores nucleares de la profesión, asumen el reto de explorar sus propias actitudes, creencias y sesgos para generar un cambio en la forma en la que se perciben a sí mismos, a las personas hacia las que va dirigida su práctica profesional y a las relaciones que mantienen con ellos. La perspectiva del empoderamiento se opone al modelo clásico del diagnóstico/ problema-patología donde, tras diagnosticar el problema, se prescribe la solución. En contraposición a este modelo, se enfatiza el crecimiento, el cambio, la colaboración e identifica a la PM como agente y protagonista del mismo ${ }^{10}$.

Desde nuestra perspectiva, el profesional debe realizar una valoración específica de todos los aspectos implicados en cada decisión para que la persona pueda conocer todo lo relacionado con ella, incluyendo potenciales riesgos y beneficios, además de asegurar el uso del lenguaje adecuado a la cultura y capacidad de cada persona ${ }^{11}$. Esta reflexión operativa requiere aprendizaje, esfuerzo, y una alta dosis de compromiso por parte del profesional ${ }^{12}$. Optimizar y facilitar los procesos de empoderamiento de las PM, máxime si se encuentran en situación de fragilidad o dependencia y/o heteronomía, constituye un eje esencial en la construcción cotidiana de relaciones e intervenciones centradas en las personas. Ello implica la revisión de las competencias específicas de los profesionales al respecto, así como la necesaria adaptación del rol e identidad profesional $^{13,14}$.

La relación entre los profesionales y las PM es una relación de apoyo o de ayuda en la que se distinguen 2 intensidades: una centrada en la promoción y otra en el cuidado según la diferente intensidad en la que se deba compensar la falta de autonomía y/o independencia de la PM. Lo idóneo será establecer, para cada persona y en cada circunstancia, la intensidad adecuada, modulándola según los cambios que se vayan produciendo. No hacerlo se puede considerar como mala praxis profesional ${ }^{1}$ ya que la determinación de la competencia de la persona y el respeto a su autonomía moral son habilidades y actitudes necesarias en la atención e intervención con $\mathrm{PM}^{15}$.

\section{Bibliografía}

1. Bermejo L. Guía de buenas prácticas en residencias de personas mayores en situación de dependencia. Oviedo: Conseyería de Bienestar Social y Vivienda del Principáu d'Asturies; 2009 [consultado 10 Jul 2014]. Disponible en: https:// www.asturias.es/Asturias/descargas/PDF_TEMAS/Asuntos\%20Sociales/Calidad/ 1.1_Residencias\%20Mayores-Parte\%20I.pdf

2. Sánchez A. Psicologia Comunitaria Bases conceptuales y métodos de intervención. Barcelona: Universidad de Barcelona; 1996.

3. Monreal-Bosch P, del Valle A. Las personas mayores como actores en la comunidad rural: innovación y empowerment. Athenea Digital. 2010;19:171-87.

4. Monreal-Bosch P, del Valle A, Perera S. Activando municipios para la promoción de la salud: un estudio de caso en comunidades rurales. Anuario de Psícología. 2013;43:217-35

5. Nussbaum M. Crear Capacidades. Barcelona: Ediciones Paidos; 2011.

6. González Menéndez R. La psicología en el campo de la salud y la enfermedad. La Habana: Editorial Científico Técnico; 2004.

7. Siegler M. The progression of medicine: From physician paternalism to patient autonomy to bureaucratic parsimony. Arch Intern Med. 1985;145:713-5.

8. Durán Muñoz R. Envejecer y empoderar, una propuesta analítica. Rev Esp Geriatr Gerontol. 2007;42:293-301

9. Weick A. Building a Strengths Perspective for Social Work. En: Saleebey D, editor. The strengths perspective in social work practice. New York: Longman; 1992. p. 18-38.

10. Hernán M, Lineros C. Los Activos para la salud. Granada: EASP; 2009

11. Sessums LL, Zembrzuska H, Jackson JL. Does this patient have medical decisionmaking capacity? JAMA. 2011;27:420-7.

12. Altisent R. La gestión de la autonomía en la práctica clínica. EIDON. 2013;39:35-43.

13. Bermejo L. Los profesionales en el modelo de Atención Integral y Centrada en la Persona. En: En AAVV: Manual de Atención Integral y Centrada en la Persona. Madrid: Fundación Pilares para la Autonomía Personal; 2014.

14. Bermejo L. Estudio sobre la Creación de una Unidad de Calidad de vida. Madrid IMSERSO: 2014

15. Alonso-Renedo FJ, González-Ercilla L, Iráizoz-Apezteguía I. El anciano con enfermedad avanzada de órgano. Consideraciones desde la geriatría, la medicina paliativa y la bioética. Rev Esp Geriatr Gerontol. 2014;49:228-34. 